\title{
BALANCED PRESENTAIONS OF THE TRIVIAL GROUP AND FOUR-DIMENSIONAL GEOMETRY
}

\author{
BORIS LISHAK AND ALEXANDER NABUTOVSKY
}

\begin{abstract}
We prove that 1) There exist infinitely many non-trivial codimension one "thick" knots in $\mathbb{R}^{5} ; 2$ ) For each closed four-dimensional smooth manifold $M$ and for each sufficiently small positive $\varepsilon$ the set of isometry classes of Riemannian metrics with volume equal to 1 and injectivity radius greater than $\varepsilon$ is disconnected; 3) For each closed four-dimensional $P L$-manifold $M$ and any $m$ there exist arbitrarily large values of $N$ such that some two triangulations of $M$ with $<N$ simplices cannot be connected by any sequence of $<M_{m}(N)$ bistellar transformations, where $M_{m}(N)=\exp (\exp (\ldots \exp (N)))$ ( $m$ times $)$.
\end{abstract}

\section{MAin RESUlts.}

1.1. The goal of this paper is to extend results of [N1], [N2], [N3] to the fourdimensional situation.

Theorem 1.1. Let $M$ be any closed four-dimensional Riemannian manifold. Let $I_{\varepsilon}(M)$ denote the space of isometry classes of Riemannian metrics on $M$ with volume equal to 1 and injectivity radius greater than $\varepsilon$. (This space is endowed with the Gromov-Hausdorff metric $d_{G H}$.) Then for all sufficiently small $\varepsilon>0 I_{\varepsilon}(M)$ is disconnected, and, moreover, can be represented at the union of two non-empty subsets $A_{1}, A_{2}$ such that for any $\mu_{1} \in A_{1}, \mu_{2} \in A_{2} d_{G H}\left(\mu_{1}, \mu_{2}\right)>\frac{\varepsilon}{10}$.

Furthermore, let for each $m \exp _{m}(x)$ denote $\exp (\exp (\ldots(\exp x)))$ ( $m$ times). Then for each $m$ for all sufficiently small $\varepsilon$ there exist $\mu, \nu \in I_{\varepsilon}(M)$ with the following property. Let $\mu_{1}=\mu, \mu_{2}, \ldots, \mu_{N}=\nu$ be a sequence of isometry classes of Riemannian metrics on $M$ of volume one such that for each $i d_{G H}\left(\mu_{i}, \mu_{i+1}\right) \leq \frac{\varepsilon}{10}$. Then $\inf _{i} \operatorname{inj}\left(\mu_{i}\right) \leq \frac{1}{\exp _{m}\left(\frac{1}{\varepsilon}\right)}$.

A (stronger) analog of this theorem for $n>4$ as well as for a class of closed fourdimensional manifolds representable as the connected sum of any closed 4-manifold and several copies of $S^{2} \times S^{2}$ can be found in [N2] (Theorem 1 and section 5.A). (More precisely, "several" means 14. The minimal number of copies of $S^{2} \times S^{2}$ required for the method of [N2] to work is equal to the number of relators in a sequence of finitely presented groups, where the triviality problem is algorithmically unsolvable; cf. [Sh1], [Sh2]). 
The next theorem is a four-dimensional analog of Theorem 11 from [N2]. For each smooth manifold $M$ define $A l_{1}(M)$ as the space of $C^{1}$-smooth Alexandrov spaces of curvature $-1 \leq K \leq 1, C^{1}$-diffeomorphic to $M$ (cf. [BN] for a definition of Alexandrov spaces with two-sided bounds on sectional curvature). A result of I. Nikolaev ([Ni]) implies that all of them are Gromov-Hausdorff limits of sequences of smooth Riemannian structures on $M$. The classical Gromov-Cheeger compactness theorem implies that all elements of $A l_{1}(M)$ are $C^{1, \alpha}$-smooth Riemannian structures on $M$ for any $\alpha \in(0,1)$. We can consider diameter as a functional on $A l_{1}(M)$.

Theorem 1.2. Let $M$ be a closed 4-dimensional manifold such that either its Euler characteristic is not equal to zero, or its simplicial volume is not equal to zero. Then diameter regarded as a functional on $A l_{1}(M)$ has infinitely many local minima. The set of values of diameter at its local minima on $A l_{1}(M)$ is unbounded.

The assumptions about $M$ imply a uniform positive lower bound for the volume of all elements of $A l_{1}(M)$. Now the Gromov-Cheeger theorem implies the compactness of sublevel sets of diam, $\operatorname{diam}^{-1}((0, x])$, on $A l_{1}(M)$ for all values of $x$. Now we see that it is sufficient to prove that there exists an unbounded sequence of values of $x$ such that the set of all smooth Riemannian structures on $M$ with $-1 \leq K \leq 1$ and diam $\leq x$ is disconnected, and, moreover, can be represented as a union of two non-empty subsets with disjoint closures. After noticing that the classical Cheeger inequality implies that for all such smooth Riemannian structures the injectivity radius will be bounded below by an explicit positive function of $x$ (that behaves as const $\exp (-3 x))$ we see that this theorem is similar to the previous one, and, in fact, has a very similar proof.

Theorem 11 in [N2] should not be confused with a much deeper and significantly more difficult main theorem in [NW1] (see also [NW2] and [W]) that does not have the assumption that a smooth manifold $M$ of dimension greater than four has either a non-zero Euler characteristic or a non-zero simplicial volume, and, therefore, one lacks an a priori uniform positive lower bound for the volumes of the considered metrics. At the moment we are not able to prove a four-dimensional analog of the main theorem of [NW1].

1.2. In order to state the next theorem define crumpledness (a.k.a ropelength) of an embedded closed manifold $X^{n}$ in a complete Riemannian manifold $Y^{n+k}$ as $\kappa\left(X^{n}\right)=$

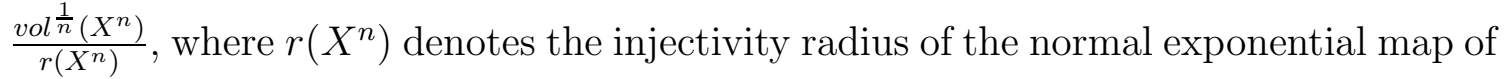
$X^{n}$. Informally speaking, $r\left(X^{n}\right)$ can be interpreted as the smallest radius of a nonselfintersecting tube around $X^{n}$. This functional was defined in [N1] for hypersurfaces and named "crumpledness", but in later papers on "thick" knots in $\mathbb{R}^{3}$ it had been given a new name "ropelength", as it can be interpreted as the length of a similar knot such that the maximal radius of a nonself-intersecting tube around this knot 
is equal to one (i.e. it is the length of a similar knot tied on "thick" rope of radius one). One of the ideas of [N1] was that one can similarly consider higher-dimensional "thick" knots. Two knots (=embeddings of $S^{n}$ in $\mathbb{R}^{n+k}$ ) belong to the same $x$-thick knot type if they both are in the same path component of the sublevel set $\kappa^{-1}((0, x])$ of $\kappa$.

To state our main result about "thick" knots it is convenient to first introduce a space of non-parametrized $C^{1,1}$-smooth embeddings $E_{n}=\operatorname{Emb}\left(S^{n}, R^{n+1}\right) / \operatorname{Diff}\left(S^{n}\right)$ of $S^{n}$ into $\mathbb{R}^{n+1}$, and then define $K_{n o t}{ }_{n, 1}$ as the quotient of $E_{n}$ with respect to the action of the group generated by isometries and homotheties of the ambient Euclidean space $\mathbb{R}^{n+1}$. The choice of smoothness is motivated by the facts that 1) $r\left(\Sigma^{n}\right)>0$ for every $C^{1,1}$-smooth closed hypersurface; 2) $r$ is an upper semi-continuous functional of $E_{n}$ and, therefore, $K_{n o t}{ }_{n, 1}$ (Theorem 5.1.1 of [N1]); and 3) The sublevel sets $\kappa^{-1}((0, x])$ in $K_{n o t}, 1$ are compact (see [N1], proof of Theorem 5.2.1). These facts are true for all dimensions $n$. Now for each $x$ we can consider "thick" knot $x$-types as subsets of either $E_{n}$ or $K$ not $_{n, 1}$. A knot $x_{1}$-type and $x_{2}$-type are distinct if they do not intersect in $E_{n}$ (or, equivalently, in $K$ not $_{n, 1}$ ). (Assuming that, say, $x_{1} \leq x_{2}$, this is equivalent to the $x_{1}$-knot not being a subset of the $x_{2}$-knot.) Our next results imply that there exist non-trivial types of "thick" four-dimensional knot types of codimension one.

Theorem 1.3. There exists an infinite sequence of distinct $x_{i}$-knot types in $E_{4}$ (correspondingly, Knot $_{4,1}$ ), where $x_{i}$ is an unbounded increasing sequence. Moreover, there exists an unbounded increasing sequence of $x_{i}$, which are the values of $\kappa$ at its local minima $k_{i}$ on $E_{4}$ (or, equivalently, $K_{n o t} t_{4,1}$ ). Further, for each $m$ one can find such a sequence of numbers $\left\{x_{i}\right\}$ and knots $k_{i}$ with the additional property that any isotopy between $k_{i}$ and the standard 4 -sphere of radius 1 in $\mathbb{R}^{5}$ must pass through hypersurfaces, where the value of $\kappa$ is greater than $\exp _{m}\left(x_{i}\right)$.

Remarks. 1. The second assertion of the theorem is stronger than the first assertion, as each local minimum of $\kappa$ with value $x$ gives rise to a $x$-knot type that consists of one knot, if the local minimum is strict, and a connected set of knots in $\kappa^{-1}(\{x\})$ otherwise. On the other hand, the second asserton immediately follows from the first assertion and the compactness of sublevel sets of $\kappa$ (see Theorem 5.1.1 in [N1]).

2. The local minima of $\kappa$ were called self-clenching hypersurfaces in [N1]. The idea behind this metaphor is that one can imagine that this hypersurface is made of very thin material that bends but cannot be stretched. If it also cannot be squeezed, then the "thick" hypersurface is tightly folded in $\mathbb{R}^{5}$. It can move (other than rigid body movement) only if the local minimum is not strict, and only by "sliding movements", so that at each moment of time it is still a local minimum of $\kappa$ (i.e. it cannot be unfolded into a less crumpled shape). 
3. Two very interesting question are whether or not there exist non-trivial "thick" knots of codimension one in $\mathbb{R}^{3}$ and $\mathbb{R}^{4}$. The second of these questions is related to the smooth Schoenflies conjecture, that asserts that each smooth embedding of $S^{3}$ into $\mathbb{R}^{4}$ is isotopic to the standard round sphere of radius one. (This fact is known for all other dimensions). Note, that if the smooth Schoenflies conjecture turns out to be false one can still ask whether or not there are non-trivial "thick" knot types in the component of $K$ not $_{3,1}$ that consists of 3 -spheres in $\mathbb{R}^{4}$ that are isotopic to the round sphere. It seems almost "self-evident" that there are no non-trivial "thick" knots $S^{1} \subset \mathbb{R}^{2}$, but we do not know a proof of this fact and are not aware of any publications in this direction.

1.3. To state our third result for every closed four-dimensional $P L$-manifold $M$ consider the set of all simplicial isomorphism classes of simplicial complexes PLhomeomorphic to $M$. For brevity, we call them triangulations of $M$. The discrete set $T(M)$ of all triangulations of $M$ can be turned into a metric space using bistellar transformations. Bistellar transformation are operations that transform one triangulation into the other as follows. Let $T_{1}$ be a triangulation of $M$. Assume that it contains a simplicial subcomplex $K$ that consists of $k, 1 \leq k \leq 5$, 4-dimensional simplices (together with their faces) and is simplicially isomorphic to a subcomplex $C$ of the boundary of a 5 -dimensional simplex $\partial \Delta^{5}$. To perform the corresponding bistellar transformation one first removes these $k$ simplices (and all their faces) and then attaches the closure of the complement $\partial \Delta^{5} \backslash C$ to the boundary of $K$ (which is simplicially isomorphic to the boundary of $\partial \Delta^{5} \backslash C$ ). Since we exchange one PL-disc (triangulated with $k$ 4-simplices) for another (triangulated with $6-k 4$-simpleces), we obtain a triangulation $T_{2}$ of the same manifold. Moreover, endow $T_{1}$ and $T_{2}$ with length metrics such that each simplex is a flat regular simplex with side length one. In this case it is easy to see that $T_{1}$ and $T_{2}$ will be bi-Lipschitz homeomorphic, and the Lipschtz constants of the homeomorphism and its inverse will not exceed an absolute constant that can be explicitly evaluated. U. Pachner proved that every two triangulations of the same closed $P L$-manifold can be connected by a finite sequence of bistellar transformations $([\mathrm{P}])$. Now one can define the distance $d_{B i s t}\left(T_{1}, T_{2}\right)$ on $t(M)$ as the minimal number of bistellar transformations required to transform $T_{1}$ into $T_{2}$.

Theorem 1.4. For each 4-dimensional closed PL-manifold $M$ and each positive integer value of $m$ there exist arbitarily large values of $N$ and two triangulations $T_{1}$, $T_{2}$ with $\leq N$ simplices such that $d_{B i s t}\left(T_{1}, T_{2}\right)>\exp _{m}(N)$. (In other words, $T_{1}$ and $T_{2}$ cannot be connected by any sequence of less than $\exp _{m}(N)$ bistellar transformations).

A stronger version of this theorem had been proven in [N3] for all manifolds of dimension greater than four as well as all four-dimensional manifolds that can be represented as a connected sum with $k$ copies of $S^{2} \times S^{2}$, where the value of $k$ can be 
chosen as 14 using [Sh1], [Sh2]. Note that results of [N3] and, especially, Theorem 1.4 for $M=S^{4}$ have potential implications for four-dimensional Euclidean Quantum Gravity (see [N4] and references there).

\section{Proofs.}

2.1. Balanced finite presentations of the trivial group. In [L] one of the authors have constructed a sequence of finite balanced presentations of the trivial group. These finite presentations have two generators and and two relations. They can be described as the Baumslag-Gersten group $B=<x, t \mid x^{x^{t}}=x^{2}>$ with an added variable second relation. (Here $a^{b}$ denotes $a b a^{-1}$.) To describe this relation note that there exists a sequence of words $v_{n}$ in $B$ of length $O(n)$ representing $x^{\left.E\left(\log _{2} n\right]\right)}$, where $E(m)$ denotes $2^{2 \cdots{ }^{2}}$ ( $m$ times). Clearly, $v_{n}$ commutes with all powers of $x$. Words $w_{n}=\left[v_{n}, x\right]$ represent the trivial element but one needs to apply the only relation at least $E\left(\left[\log _{2} n\right]-\right.$ const) times to establish this fact. (Thus, the Dehn function of the one relator group $B$ grows faster than any tower of exponentials of a fixed height of $n$, cf. $\quad[\mathrm{Ge}],[\mathrm{Pl}]$.) The extra relation added to $B$ is $\left[v_{n}, x^{3}\right]\left[v_{n}, x^{5}\right]\left[v_{n}, x^{7}\right]=t$, where $[a, b]$ denotes the commutator $a b a^{-1} b^{-1}$. The most important property of this sequence of groups is that any representation of either $x$ or $t$ as a product of conjugates of the two relators and their inverses will require at least $E\left(\left[\log _{2} n\right]-2\right)$ multipliers. Also, note that these finite presentations satisfy the Andrews-Curtis conjecture. The importance of the last observation is in the fact that when one constructs a representation complex $K$ of such a finite presentation (that is, a 2complex with one 0-dimensional cell, two 1-dimensional cells corresponding to the generators and two 2-dimensional cells corresponding to the relators), embeds it in $\mathbb{R}^{5}$, takes the boundary of a small neighborhood of the embedding, and smoothes it out, one obtains not merely a smooth homotopy 4-sphere that must be homeomorphic to $S^{4}$ by virtue of Freedman's proof of the 4-dimensional Poincare conjecture, but a manifold that is diffeomorphic to $S^{4}$. This fact can be demonstrated without the 4-dimensional Poincare conjecture using instead the fact that the operations in the Andrews-Curtis conjecture correspond to certain diffeomorphisms of the underlying manifold ("handle slidings"). A sequence of these diffeomorphisms corresponding to handle slides will eventually result in the standard sphere that corresponds to the representation 2-complex of the trivial finite presentation of the trivial group (cf. $[\mathrm{BHP}])$.

The resulting smooth hyperspheres in $\mathbb{R}^{5}$ that will be denoted by $S^{4}\left(v_{n}\right)$ can, after rescaling, be interpreted as elements of $I_{\varepsilon_{n}}\left(S^{4}\right)$ (for an appropriate $\varepsilon_{n}$ ) or $A l_{1}\left(S^{4}\right)$. We can also interprete them as elements of $K_{n o t} t_{4,1}$ or $E_{4}$. Finally, we can construct a hypersphere triangulated into flat simplices (instead of a smooth hypersphere). It is easy to see that the number of simplices will grow linearly with the length of 
the word $v_{n}$ in the Baumslag-Gersten group that was used to construct, first, the balanced finite presentation of the trivial group and, then, a 4-dimensional sphere. Similarly, in the smooth case, $\frac{v o l^{\frac{1}{4}}}{i n j}, \frac{v o l^{\frac{1}{4}}}{r}$ and $|K|$ diam $^{2}$ will be bounded above by an exponential function of const $n$ for some const (in fact, one can ensure much better bounds, but we do not need this).

These hypersurfaces in $\mathbb{R}^{5}$ constructed using the balanced presentations of the trivial group introduced in [L] will be used in the proofs of all our results. But note that in this construction one can alternatively use another family of balanced presentations of the trivial group with similar properties that were independently discovered by Martin Bridson. We were not aware of his work until this paper had almost been finished. But after [L] appeared on the arXiv Bridson e-mailed to us and wrote that he found such finite presentations in 2003. Although they were mentioned in his ICM-2006 talk ([B], p. 977), he has never publshed or posted any details of his construction on the internet. Two weeks after the appearance of [L] his preprint [B2] has also appeared on the arXiv. Note that in his ICM-2006 talk Bridson expresses a hope that such finite presentations of the trivial group can be used to extend results of Nabutovsky and Weinberger on the sublevel sets of diameter on moduli spaces to dimension 4 (which is something that we were not yet able to accomplish). So, his work [B2] was also partially motivated by potential applications that are similar in spirit to our results in this paper. Even earlier, in the $90 \mathrm{~s}$, the second author attempted to prove the results of this paper using balanced finite presentations of the trivial group obtained from $B$ in the most obvious way, namely, by adding the second relation $w_{n}=t$. Yet he was not able to verify that these balanced presentations have the desired properties.

2.2. The filling length. Following [N2] we are going to use the following characteristic of simply-connected closed Riemannian manifolds that measures how "difficult" is to contract closed curves. We define it as the supremum over all closed curves $\gamma$ of the ratio $\frac{f l(\gamma)}{\text { length }(\gamma)}$, where the filling length $f l(\gamma)$ denotes the infimum over all homotopies $H=\left(\gamma_{t}\right)_{t \in[0,1]}, \gamma_{0}=\gamma$, contracting $\gamma$ to a point (=constant curve) $\gamma_{1}$ of the maximal length $\sup _{t}$ length $\left(\gamma_{t}\right)$ of the closed curves arising during the homotopy $H$. We are going to denote this quantity by $F l$ and regard it as a functional on a considered space of (isometry classes) of Riemannian metrics.

To see that $F l<+\infty$ first note that all sufficiently short curves $\gamma$ can be contracted to a point without length increase (and, therefore, $f l(\gamma)=$ length $(\gamma)$ ). On the other hand for long curves $\gamma$ we can choose any point $z$, and connecting $z$ with a sequence of sufficiently close points on $\gamma$ by minimal geodesics reduce contraction of $\gamma$ to consecutive contractions of triangles formed by a very short arc of $\gamma$ and two minimal geodesics betwen $z$ and two very close points on $\gamma$. Perimeters of these triangles are bounded by $2 d+\varepsilon$, where $d$ is the diameter of the Riemannian manifold and $\varepsilon$ is 
arbitrarily small. This easily implies that $\frac{f l(\gamma)}{\text { length }(\gamma)} \longrightarrow 1$ as length $(\gamma) \longrightarrow \infty$. This fact was first noticed by M. Gromov $([\mathrm{Gr}])$. (The existence of the supremum for closed curves of length $\leq 2 d+\varepsilon$ follows from the compactness of the set of Lipschitz curves of length $\leq 2 d+\varepsilon$ parametrized by the arclength.) Gromov also introduced the term "filling length" and the notation $f l$ (with a slightly different meaning than what we use here).

Note that $\mathrm{Fl}$ can also be defined for all simply-connected length spaces such that for some positive $\varepsilon$ all closed curves of length $\leq \varepsilon$ can be contracted to a point without length increase. So, in particular, we can consider $F l$ as a functional on the spaces of triangulations of closed manifolds after we endow each simplex of the maximal dimension by the metric of a regular flat simplex with side length 1. (Actually, it is easy to see that $F l$ will not depend on the choice of side length here.)

Our observation is that if $S^{4}\left(v_{n}\right)$ is a (smooth or PL) sphere: constructed starting from the word $v_{n}$ in the Baumslag-Gersten group as expalined above (using either the idea from $[\mathrm{L}]$ or the idea from $[\mathrm{B} 2])$ then:

Proposition 2.1. The value of $F l_{n}=F l\left(S^{4}\left(v_{n}\right)\right)$ grows faster than any finite tower of exponentials of $n$.

Proof. Indeed, if not, then we can prove that the area of van Kampen diagrams for generators of $S^{4}\left(v_{n}\right)$ will also be bounded by towers of exponentials of $n$ of a fixed height. In the proof below we use the same notation const for different constants that can be, in principle, evaluated.

The idea is that one can choose a way to represent each close curve $\gamma$ of length $\leq x$ by a word of length $\leq$ const $n x$ so that if two closed curves $\gamma_{1}$ and $\gamma_{2}$ are const-close, then the corresponding words can be connected by a sequence of at most const $x$ relations. In order to achieve this we first project $\gamma$ to the embedding of the representation complex of the balanced presentation in $\mathbb{R}^{5}$. Recall, that $S^{4}\left(v_{4}\right)$ is the smoothed-out boundary of a small tubular neighborhood of the representation complex, so this step will increase the length by at most const $n$ factor. Denote the projection of $\gamma$ to the embedded representation complex by $\tilde{\gamma}$.

Note that if $D$ is a Riemannian 2-disc one can choose a way to replace each arc with endpoints on $\partial D$ by a shortest arc of $\partial D$ with the same endpoints. An ambiguity in the situation when the distance between the endpoints along the boundary is equal to $\frac{|\partial D|}{2}$ leads to a discontinuity, and arcs corresponding to two very close curves can together almost form the boundary of $D$. Consider now one of the two 2-cells in the representation complex and a connected smooth arc $A$ in its interior with end points on its boundary. The boundary of the 2-cell has some self-intersections that appeared as the result of taking the quotient map, when the cell was attached to the 1-skeleton. Yet we can canonically lift $A$ to the 2-disc with the same Riemannian metric in the interior and with nonself-intersecting boundary, then extend $A$ to the 
boundary, replace it by a shortest arc of the boundary with the same endpoints, and, finally, project this arc back to the 1-skeleton of the embedded representation complex.

Now take each component of the intersection of $\tilde{\gamma}$ with $e_{i}, i=1,2$ and replace it by a minimal arc in the boundary of $\partial e_{i}$ with the same endpoints as explained above. This will result in a length increase by at most const $n$ factor. The mentioned ambiguity in the case when the points of intersection with $\partial e_{i}$ can be connected in the lift of $\partial e_{i}$ by two arcs of length $\frac{\partial e_{i}}{2}$ is a source of discontinuity of this process. If such a discontinuity arises for a pair of close curves $\gamma_{1}, \gamma_{2}$, then the resulting arcs in the 1-skeleton of the representation complex will (almost) form the boundary of $e_{i}$. Also, note that such ambiguity (discontinuity) can arise only for a sufficiently long arc of $\gamma \bigcap e_{i}$, and, therefore, the number of such occurences for a closed curve $\gamma$ is bounded by const $x$. Now note that once $\gamma$ is replaced by a closed curve in the 1-skeleton of the representation complex, we can assign to it a word, and conclude that these words for sufficiently close curves can be transformed one into the other by an application of at most const $x$ relations.

Now we observe that a well-known and easy argument implies that for each $\delta>0$ there exists a $\delta$-net in the space of closed curves of length $\leq x$ in the constructed Riemannian manifold of cardinality bounded by $\exp \left(\right.$ const $\left.\frac{x}{\delta}\right)$. Therefore, each closed curve can be contracted to a point by a discretized homotopy that consists of at most $\exp ($ const $x)$ "jumps" of "length" $\leq$ const so that each "jump" corresponds to a sequence of not more than const $x$ applications of the relations for words corresponding to the curves.

Finally, let $\gamma_{0}$ be a closed curve that represents one of the generators of the considered finite presentation. It can be connected to a point through curves of length $\leq F l_{n}$ length $\left(\gamma_{0}\right)$. Therefore, one obtains an at most exponential in const $F l_{n}$ upper bound for the number of the relations required to demonstrate that the generator, is, indeed, trivial. This completes the proof of the proposition.

Let $M_{0}$ be any closed simply connected 4-dimensional Riemannian manifold. We can form a Riemannian connected sum of $M_{0}$ with the spheres $S^{4}\left(v_{n}\right)$ in an obvious way and observe that $F l$ for resulting Riemannian manifolds grows faster than any tower of exponentials of $n$ of a fixed height.

2.3. Proof of theorems. It is now easy to prove the main theorems for simply connected manifolds. In order to prove Theorem 1.4 recall that each bistellar transformation leads to a bi-Lipschitz homeomorphism of the underlying simplicial complexes regarded as metric spaces, where each face of dimension four is given the metric of the regular 4-simplex with the side length 1. The Lipschitz constants for the map and its inverse do not exceed an absolute constant const. Now note that in this situation $F l$ cannot change by more than the factor const ${ }^{2}$. The value of $F l$ for the boundary $\partial \Delta^{5}$ of the regular 4 -simplex (endowed with the standard metric) is 1 . 
Therefore, the value of $F l$ on each triangulation of $S^{4}$ that can be connected with $\partial \Delta^{5}$ by at most $M$ bistellar transformation is at most const ${ }^{2 M}$. This fact immediately implies the assertion of the theorem.

The proofs of the first three theorems are similar. The idea is to prove that if the assertion does not hold, then $F l_{n}$ is bounded above by a tower of exponentials of $n$ of a fixed height, and this would contradict the assertion of Proposition 2.1.

One can follow [N2] to finish the proof of Theorem 1.1. One starts from the observation that if two Riemannian structures in $I_{\varepsilon}(M)$ are $\frac{\varepsilon}{8.5}$-close (in the GromovHausdorff metric), then the values of $F l$ can differ by a factor that does not exceed 1000000 (Lemma 2 in [N2]). (The idea is that if $M_{1}$ and $M_{2}$ are close Riemannian manifolds and one can contract any closed curve in $M_{2}$ through not too long curves, one can try to contract any closed curve $\gamma$ in $M_{1}$ by 1 ) discretizing it, moving points to the closest points in $M_{2}$ and connecting them by minimal geodesics, thereby obtaining a closed curve $\gamma_{2}$ that can be regarded as a "transfer" of $\gamma$ to $M_{2} ; 2$ ) Contracting $\gamma_{2}$ through not too long curves in $M_{2} ; 3$ ) Discretizing this homotopy and transfering closed curves in the discretization back to $\left.M_{1} ; 4\right)$ Connecting the transfers of the nearest closed curves by homotopies in $M_{1}$, thus, obtaining a homotopy contracting $\gamma$ in $M_{1}$.)

The second observation used in [N2] is that one can use the well-known proof of the fact that $I_{\varepsilon}(M)$ is precompact to give an explicit uper bound of the form $\exp \left(\frac{\text { const }}{\varepsilon^{9}}\right)$ (in the four-dimensional case) for the cardinality of an $\varepsilon / 20$-net in $I_{\varepsilon}(M)$. This estimate can then be used to conclude that any two Riemannian structures in the same connect component of $I_{\varepsilon}(M)$ can be connected by a sequence of $\varepsilon / 9$-long "jumps", so that the number of jumps does not exceed $\exp \left(\frac{\text { const }}{\varepsilon^{9}}\right)$ (see the proof of Lemma 3 in [N2]). Combining this estimate with the previous observation we see that the ratio of values of $F l$ at any two elements of the same connected component of $I_{\varepsilon}(M)$ is bounded by a double exponential of a power of $\frac{1}{\varepsilon}$ (and, thus, by a triple exponential function of $\frac{1}{\varepsilon}$ for all sufficiently small values of $\varepsilon$ ). This estimate can be generalized to a stronger equivalence relation on $I_{\varepsilon}(M)$ than being in the same connected component, namely, the transitive closure of the relation "to be $\frac{\varepsilon}{9}$-close in the Gromov-Hausdorff metric".

A comparison of these triply exponential upper bounds with lower bounds for $F l$ that grow faster than any tower of exponential of a fixed height of $n$ yields the assertion of Theorem 1.1.

As it had been noticed, Theorem 1.2 would follow from the disconnectedness of sublevel sets of the diameter $\operatorname{diam}^{-1}((0, x])$ on $A l_{1}(M)$, and the injectivity radius is bounded below by $\exp (-$ const $x)$ on these sets. Now one can use the same argument as in the proof of Theorem 1.1. 
To prove Theorem 1.3 we can rescale the hypersurface to have the value of the volume equal to 1 . Now note that the definition of $\kappa$ implies that $\kappa \geq|k|$, where $k$ denotes any of the principal curvatures of the hypersurface. This implies the obvious upper bound for the absolute values of its sectional curvatures, when it is regarded as a Riemannian manifold. It is not difficult to establish an upper bound for the diameter of the hypersurface in the inner metric (which immediately follows from Theorem 1.1 in $[\mathrm{T}]$ ). Now the Cheeger inequality implies an explicit lower bound for the injectivity radius of the hypersurface that behaves as $\exp \left(-\right.$ const $\left.\kappa^{\text {const }}\right)$, and we can prove the disconnectedness of sublevel sets of $\kappa$ for an unbounded sequence of values of $x$ exactly as we proved the disconnectedness of $I_{\varepsilon}(M)$.

The proofs of Theorems 1.1, 1.2 and 1.4 in the case of a nonsimply connected manifold can be based on the same ideas. We form a Riemannian connected sum of $M$ endowed with some Riemannian metric with $S^{4}\left(v_{n}\right)$. Now $F l$ is not defined, but we can look at how much the length of the closed curves corresponding to the generators of the balanced presentations must be increased before they can be contracted to a point. An argument similar to the proof of Proposition 2.1 implies that the growth of this quantity with $n$ is faster than any tower of exponentials of a fixed height. On the other hand the arguments in this section can be used to demonstrate that the connectedness assumptions imply a much slower growth of this quantity.

Acknowledgements. This work has been partially supported from NSERC Accelerator and Discovery Grants of second named author (A.N.).

\section{References.}

[BN] V. Berestovskii, I. Nikolaev, "Multidimensional generalized Riemannian spaces", in "Geometry iV", ed. Yu. G. Reshetnyak, Encyclopedia of Mathematical Sciences, vol. 70, Springer, 1993.

[BHP] W. Boone, W. Haken, and V. Poenaru, "On Recursively Unsolvable Problems in Topology and Their Classification", Contributions to Mathematical Logic (H.

Arnold Schmidt, K. Schütte, and H. J. Thiele, eds.), North-Holland, Amsterdam, 1968.

[B] M. Bridson, "Non-positive curvature and complexity for finitely presented groups", Proceedings of the ICM, Madrid, Spain, 2006, 961-987, European Mathematical Society, 2006.

[B2] M. Bridson, "The complexity of balanced presentations and the Andrews-Curtis conjecture", arXiv:1504.04261

[Ge] S. M. Gersten, Dehn functions and $l_{1}$-norms of finite presentations, Algorithms and Clas- sification in combinatorial group theory (Berkeley, CA, 1989) (G. Baumslag, C. Miller, eds.), Math. Sci. Res. Inst. Publ. 23, Springer-Verlag (1992), 195-224.

[Gr] M. Gromov, "Metric structures for Riemannian and non-Riemannian spaces", Birkhauser, 1998. 
[L] B. Lishak, "Balanced finite presentations of the trivial group", arXiv:1504.00418. [N1] A. Nabutovsky, "Non-recursive functions, knots "with thick ropes", and selfclenching "thick" hypersurfaces", Comm. Pure Appl. Math. 48(1995), 381-428.

[N2] A. Nabutovsky, "Disconnectedness of sublevel sets of some Riemannian functionals", Gem. Funct. Analysis (GAFA), 6(1996), 703-725.

[N3] A. Nabutovsky, "Geometry of the space of triangulations of a compact manifold", Comm. Math. Phys. 181(1996), 303-330.

[N4] A. Nabutovsky, "Combinatorics of the space of Riemannian structures and logic phenomena of Euclidean Quantum Gravity", in "Perspectives in Riemannian Geometry", ed. by V. Apostolov et al., CRM Proceedings and Lecture Notes, vol. 40, 223-248, AMS, Providence, RI, 2006.

[N5] A. Nabutovsky, "Morse landscapes of Riemannian functionals and related problems", Proceedings of ICM-2010, vol. 2, 862-881, Hindustan Book Agency, New Delhi, 2010.

[NW1] A. Nabutovsky, S. Weinberger, "Variational problems for Riemannian functionals and arithmetic groups", Publications d'IHES, 92(2000), 5-62.

[NW2] A. Nabutovsky, S. Weinberger, "The fractal nature of Riem/Diff I", Geom. Dedicata 101(2003), 1-54.

[Ni] I. Nikolaev, "Bounded curvature closure of the set of compact Riemannian manifolds", Bull. of the AMS 24(1992), 171-177.

[M] J. Milnor, "Lectures on the $h$-cobordism theorem", Princeton University Press, Princeton, NJ, 1965.

[P] U. Pachner, "P.L. homeomorphic manifolds are equivalent by elementary shellings", Europ. J. Combinatorics 12(1991), 129-145.

[Pl] A. N. Platonov An isoperimetric function of the Baumslag-Gersten group, Moscow Univ. Math. Bull. 59 (2004), no 3, 12-17.

[Sh 1] M. A. Stan'ko "On the Markov theorem on algorithmic nonrecognizability of manifolds", J. of Mathematical Sci., 146(2007), 5622-5623.

[Sh 2] M. A. Stan'ko "The Markov theorem and algorithmically unrecognizable combinatorial manifolds", Izv. Akad. Nauk SSSR, Ser. Math., 68(2004), 207-224.

[T] P. Topping, "Relating diameter and mean curvature for submanifolds of Euclidean space", Comment. Math. Helv. 83(2008), 539-546.

[W] S. Weinberger, "Computers, rigidity and moduli", Princeton University Press, 2004. 